Die Diskussion «neuer Modelle in der medizinischen Grundversorgung» wird auch in der Schweiz seit einiger Zeit geführt. Fand sie zunächst eher in spezialisierten Kreisen und «hinter den Kulissen» statt, werden entsprechende Ideen und Vorstellungen zunehmend einem breiteren Publikum vorgestellt, auch in der SÄZ. So hat die Schweizerische Akademie der Medizinischen Wissenschaft in der SÄZ Nr. 46/2007 den Bericht ihrer Arbeitsgruppe Berufsbilder publiziert und damit auch signalisiert, dass die Ärzteschaft bei Weichenstellungen in einem Bereich, der ihre vitalen Interessen tangiert, eine aktive Rolle spielen möchte. Der folgende Beitrag, der sich spe- zifisch mit der Frage der Einführung von Nurse practitioners in der Schweiz auseinandersetzt, stammt dagegen aus der Feder eines Pflegewissenschaftlers. Aus der Sicht des Autors würde die Einführung von Nurse practitioners in der Schweiz zu einer Art «Win-win-Situation» führen: einer Stärkung der Hausarztmedizin, von der Ärzteschaft und Pflegende, vor allem aber die Patienten profitieren würden. Eine Einschätzung, die sicher nicht nur auf Zustimmung stossen wird - wir sind auf Reaktionen und weitere Beiträge gespannt.

Die Redaktion

\title{
Nurse practitioners in der medizinischen Grundversorgung der Schweiz?*
}

Chris Gut

Doktorand klinische Pflegewissenschaft, University of Bath/England

\footnotetext{
* Beim Artikel handelt es sich um die deutsche Zusammenfassung einer vom Autor verfassten Projektarbeit mit dem Titel «History and development of the nurse practitioner: Expanding the boundaries of primary healthcare provision with the implementation of advanced nursing practice in Switzerland».
}

Korrespondenz:

Chris Gut MSc

Rettungsdienst Spital Uster

Brunnenstrasse 42

CH-8610 Uster

Tel. 0449111161

chris.gut@spitaluster.ch

\section{Einleitung}

In einem Land mit Spitzenmedizin hat sich die Bevölkerung an eine effektive und prompte medizinische Grundversorgung gewöhnt. Die Hausärzte bilden die Grundpfeiler dieser Dienstleistung, in ländlichen wie auch in städtischen Gebieten. In den letzten Jahren hat die Schweiz medizinisch eine organisatorische und finanzielle Umstrukturierung erlebt: Der finanzielle und politische Druck hinterlässt Spuren in der ärztlichen Versorgung unseres Landes, die bis jetzt von moderner Technologie, solider Krankenversicherung, staatlichen Zuschüssen und ausgezeichneter Infrastruktur profitieren konnte.

Die ärztliche Primärversorgung trägt eine grosse Last in Anbetracht der zunehmenden gesellschaftlichen, finanziellen und politischen Erwartungen. Der Druck, alternative Modelle der medizinischen Grundversorgung zu suchen, steigt stetig, da der Beruf des Hausarztes immer unattraktiver wird und Nachwuchsprobleme vorprogrammiert sind.

Medizinische Fakultäten und Fachgesellschaften versuchen mit verschiedenen Strategien die Bedeutung der Allgemeinmediziner zu stärken, um einer möglichen Unterversorgung durch Hausärzte entgegenzuwirken und Nachwuchsprobleme zu beheben. Dass diese Massnahmen den drohenden Ärzteschwund in der Primärversorgung beheben können, ist unwahrscheinlich.

Meyer [1] beschreibt Pläne für die Entwicklung und Einführung von Nurse practitioners in vorwiegend ländlichen Gebieten der Schweiz. Gleichzeitig wird gemäss Meyer das hohe Interesse an «advanced nursing practice» z.B. von
Kohler [2] kritisiert, der die mit diesem Modell verbundene Möglichkeit einer Linderung des zunehmenden Drucks auf die Allgemeinmediziner ausklammert und eine ausschliesslich ärztliche Grundversorgung befürwortet. Obwohl solche Artikel subjektiver Natur sind, widerspiegeln sie einen gewissen Widerstand der Schweizer Ärzteschaft, festgefahrene und traditionelle Strukturen zu ändern und neue Wege in der medizinischen Grundversorgung einzuschlagen. Entwicklung und Ausbau von selbständigen Rollen in der Pflege werden erschwert, da standespolitische und emotionale Aspekte einen sinnvollen Dialog behindern: Die Pflege möchte sich im Rahmen der zunehmenden Akademisierung und Professionalisierung ihres Berufes weiterentwickeln, und Ärzte fühlen sich in ihren Kompetenzen von der Öffentlichkeit und Politik zunehmend unterschätzt und eingeschränkt [3].

Dieser Artikel soll eine sachliche Diskussion über die Entwicklung von «advanced nursing practice» in der Schweiz fördern und über eine neue Möglichkeit der ärztlich-pflegerischen Zusammenarbeit in der medizinischen Grundversorgung informieren.

\section{Rollendefinitionen}

Die verschiedenen Titel, die momentan aus dem angelsächsischen Raum stammen, sind gewöhnungsbedürftig. Bis anhin waren in der Schweiz spezialisierte Pflegefachkräfte nur in einzelnen Bereichen wie z.B. Anästhesiepflege, Geburtshilfe (Hebammen) usw. bekannt. Die Bezeichnungen advanced practice nurses, clinical nurse specialists, nurse consultants und nurse practitioners stammen aus vorwiegend englischsprachigen 
Ländern wie den USA, England und Australien, wo spezialisierte oder ärztlich delegierte Leistungen von Fachpflegern durchgeführt werden. Technologische und wissenschaftliche Fortschritte in der Medizin sowie die verbreitete Möglichkeit, auf Masterniveau zu studieren, werden als Gründe für diese Entwicklung der Pflege bezeichnet [4]. Leutenegger [5] betrachtet die Akademisierung der Pflege als Vorstoss in Richtung einer veränderten Gesundheitspolitik in der Schweiz.

Der «Council of the Royal College of Nursing in England» definiert den Nurse practitioner als eine Pflegefachperson, die selbständig und in eigener Verantwortung diagnostische und therapeutische Entscheidungen fällt. Dies beinhaltet auch Leistungen, die sonst von Ärzten ausgeführt werden, wie z.B. medizinische Beurteilungen, Gesundheitschecks, Krankheitsprävention und Gesundheitsvorsorge. Der Nurse practitioner erfüllt ebenfalls eine wichtige Triagefunktion und überweist komplexere Fälle an einen Arzt oder andere medizinische Institutionen. Obwohl der Nurse practitioner den Arzt keineswegs ersetzen kann und darf, muss davon ausgegangen werden, dass er für seine Leistungen die medizinische Verantwortung tragen muss.

Die rechtliche Handhabe ist diesbezüglich sehr komplex. Die organisatorischen und strategischen Veränderungen in der Pflege haben die Entwicklung neuer Rollen beschleunigt, um Erwartung und Versorgungsnotwendigkeit einer zunehmend alternden und kränklichen Gesellschaft zu begegnen. Die rechtlichen und ethischen Aspekte werden durch eigenständiges medizinisches Handeln und neue Kompetenzen beeinflusst [6]. Dafür müssen Rahmenbedingungen auch in der Schweiz definiert und entwickelt werden. In Nordamerika müssen Nurse practitioners eigene Rechtsschutz- und Haftpflichtversicherungen abschliessen und sind an strenge Lizenz- und Weiterbildungsregelungen gebunden, um praktizieren zu dürfen.

\section{Akademische Entwicklung der Pflege in der Schweiz}

Die akademische Entwicklung der Pflege in der Schweiz ist im Wachstum begiffen und verändert die Pflegelandschaft eines Landes, das bis jetzt fast ausschliesslich klassische Pflegerollen und Ausbildungen gekannt hat [7]. Die Einführung von advanced nursing practice beschränkt sich momentan auf den Bereich der chronischen Krankheiten, ähnlich wie die Funktion eines «clinical nurse specialist» (klinischer Fachpfleger). Im Gegensatz zum Nurse practitioner ist der klinische Fachpfleger ein spezialisierter Pfleger,

\section{Des «nurse practitioners»}

\section{en médecine de premier recours}

\section{en Suisse?}

Dans l'espace anglo-saxon, les prestations de médecine de premier recours sont de plus en plus données par un personnel non médical. Une telle évolution est également discutée en Suisse. Le besoin grandissant de médecins de premier recours suite au vieillissement de la population laisse clairement entendre l'appel à de nouveaux modèles dans les soins médicaux de base, car le nombre de médecins de famille diminue et des goulets d'étranglement apparaissent aujourd'hui déjà. Différentes études montrent que le personnel infirmier formé à l'université (nurse practitioners) et bénéficiant d'une formation postgraduée médicale approfondie au niveau du master ou du doctorat permet des soins de premier recours de bonne qualité et décharge les médecins de famille. Mais une telle évolution déclenche en Suisse des discussions de politique professionnelle et des peurs existentielles chez les médecins de famille, qui doivent être prises au sérieux. L'intégration de nurse practitioners dans les soins médicaux de premier recours n'empêchera pas la promotion et le renforcement de la médecine de famille. Au contraire, une collaboration partenariale garantira, à l'avenir, des soins médicaux de haute qualité car le besoin grandissant de soins médicaux nécessitera du personnel supplémentaire.

der in einer klinischen Umgebung ärztliche Anordnungen auf hohem Niveau umsetzt, die Diagnose und Therapie liegt aber in der Verantwortung des Arztes. Eine weiter gehende, selbständige Tätigkeit wie die des Nurse practitioner wird durch den Wegfall der medizinischen Komponente in der Pflegeausbildung der letzten 30 Jahre erschwert. Die Fokussierung auf soziologische und anthropologische Konzepte hat die Unterschiede in der medizinischen und der pflegerischen Ausbildung zu weit auseinandergetrieben [7]. Die akademische Pflegeausbildung muss deshalb grundlegend angepasst und erweitert werden, um die Rolle des Nurse practitioner in der Schweiz zu ermöglichen. 
Das Institut für Pflegewissenschaft INS an der Universität Basel und Schweizer Fachhochschulen bieten Studiengänge in Pflegewissenschaft an [8]. Die Einführung von Pflegeausbildungen auf Masterstufe könnte den Weg für die Einführung von erweiterten Pflegerollen wie die des Nurse practitioner ebnen. Die Umsetzung der Bologna-Reform an Schweizer Universitäten wird diese Entwicklung beschleunigen, da spezialisierte Pfleger für das selbständige Praktizieren mindestens einen Master-Abschluss vorweisen sollten. In den USA und in England sind Bestrebungen in Gange, wonach Nurse practitioners sogar einen Doktortitel erwerben können sollen.

Obwohl die in der Schweiz durchgeführten Masterstudiengänge klinische Komponenten beinhalten, müsste die Ausbildung erweitert werden. Dazu würden ein klinisches Praktikum unter der direkten Supervision eines Arztes sowie eine fundierte Vertiefung in den Bereichen Medizin, Pathophysiologie und Pharmakologie gehören. Diese Zusatzausbildung könnte in Zusammenarbeit mit den medizinischen Fakultäten angeboten werden, obwohl seitens Pflege wie auch seitens der Ärzte mit Widerstand gerechnet werden muss. Nissen-Druey [9] bezeichnet eine solche Strategie als sinnvolle Ressourcennutzung, die die Zusammenarbeit zwischen Pflege und Medizin verbessern würde. Die emeritierte Professorin des Institutes für Pathophysiologie an der Universität Basel sieht die Möglichkeit von gemeinsamen Vorlesungen von Pflege- und Medizinstudenten als eine Attraktivitätssteigerung für den Pflegeberuf. Solche kombinierten Lehrgänge würden die Qualität der selbständigen Pflegerollen erhöhen und das oftmals in effiziente Hierarchiedenken abbauen, das eine optimale Zusammenarbeit in klinischen Einrichtungen erschwert.

\section{Qualitätsvergleich zwischen Nurse practitioners und Hausärzten}

Das Konzept, Fachpfleger an der Front der medizinischen Grundversorgung im Hausarzt- und Notfallbereich einzusetzen, gewinnt im englischsprachigen Raum immer mehr an Interesse [10]. Die professionellen Grenzen und Rollendefinitionen werden neu gesteckt, da akademisch ausgebildete Pflegekräfte Tätigkeiten ausüben, die traditionell durch Ärzte getätigt wurden. Diese Entwicklung wird auch im britischen «National Health Service» (NHS) umgesetzt, wo viele medizinische Dienstleistungen wie Kliniken, Notfalldienste, Telemedizin und Medikamentenverschreibungen durch geeignet weitergebildete Pflegefachkräfte ausgeführt werden [11].
Qualitäts- und Kostenfaktoren haben einen grossen Einfluss auf die Entwicklung und Einbindung von Nurse practitioners. Obwohl Flanagan [12] die Rolle des Nurse practitioner als gute Alternative zur ärztlichen Versorgung bezeichnet, warnt er vor überhöhten Erwartungen und weist ausdrücklich auf die Tatsache hin, dass Nurse practitioners wohl sehr kompetent einfache Fälle behandeln können, aber komplexe Gesundheitsstörungen unbedingt in ärztliche Hände gehören. Einige Studien zeigen aber, dass Patienten sehr zufrieden oder sogar zufriedener sind mit ihrer Behandlung durch Nurse practitioners und dass die Behandlungskosten teilweise etwas tiefer sind [13]. In der Schweiz müsste dieser Aspekt gründlich untersucht werden, da spezialisierte Pfleger ähnliche Lohnkosten verursachen wie z.B. Assistenzärzte.

Eine Kombination von Nurse practitioners und Ärzten in der medizinischen Grundversorgung könnte allen Beteiligten, vor allem den Patienten, zugute kommen. Studien zeigen eine mögliche Kostenoptimierung und Qualitätssteigerung in Notfallzentren, Pflegeheimen und anderen klinischen Institutionen. Eine solche Zusammenarbeit könnte auch in der Hausarztmedizin zu erhöhter Arbeitszufriedenheit und weniger Arbeitsbelastung führen, da der Arzt seine Expertise vermehrt für komplexere Fälle einsetzen könnte, um sich somit von Routinearbeiten zu entlasten [13].

\section{Barrieren bei der Einführung einer Nurse-practitioner-Rolle}

Neuerungen in einem Gesundheitssystem wird oft mit Widerstand begegnet, und die Thematik der Nurse practitioners wird in der Schweiz kontrovers diskutiert. Ihre Einführung in der medizinischen Grundversorgung würde langfristig den Abschied von einem traditionellen Versorgungsmodell bedeuten. Mit einer solchen Perspektive verbundene standespolitische und auch existentielle Ängste könnten eine solche Einführung behindern. Die Anliegen der ärztlichen Grundversorger müssen ernst genommen werden, denn die Einbindung von Nurse practitioners darf die Förderung und Stärkung der Hausarztmedizin nicht im Weg stehen.

Viele Ärzte sind der Meinung, dass die medizinische Grundversorgung aus Qualitätsgründen ausschliesslich in ärztlichen Händen liegen muss, auch wenn Studien auf viele Vorteile einer Nurse-practitioner-Rolle hinweisen [15]. Entwicklungen in anderen Ländern zeigen, dass die Unterstützung durch und die Zusammenarbeit mit Ärzten in politischen und fachlichen Belan- 
gen eine sehr grosse Bedeutung bei der Einführung von Nurse practitioners hat.

\section{Zukunftsaussichten}

In vielen Ländern haben sich Nurse practitioners als sichere und geeignete Leistungserbringer in der medizinischen Grundversorgung durchgesetzt. Oft werden sie als eine effektive, patientenfreundliche und populäre Alternative wahrgenommen und ermöglichen Ärzten, sich um komplexere Gesundheitsprobleme zu kümmern [16]. Dies ist aber nur möglich, wenn Pflegefachkräfte sich fundiert weiterbilden und sich ihr Fachwissen auf Master- oder Doktoratstufe aneignen, damit sie seitens der Ärzte wie auch der Öffentlichkeit anerkannt werden [14]. Die zukünftigen demographischen Entwicklungen und der steigende Bedarf an Primärversorgern werden den Druck in Richtung eines Umdenkens und einer Neuausrichtung der Gesundheitsversorgung in der Schweiz erhöhen. Die Zuständigkeiten der Pflege und Medizin werden zunehmend zusammenwachsen. Um Konflikte möglichst $\mathrm{zu}$ vermeiden, sollte eine partnerschaftliche Entwicklung angestrebt werden. Akademisch ausgebildete Pflegefachkräfte werden Ärzte nie ersetzen können. Sie könnten aber mit einer fundierten klinischen und akademischen Weiterbildung im Rahmen einer sinnvollen Zusammenarbeit einen bedeutenden Beitrag zur qualitativ hochstehenden medizinischen Grundversorgung unseres Landes in den nächsten Jahrzehnten leisten.

\section{Literatur}

1 Meyer P. Der Nutzen der Akademisierung von Gesundheitsberufen. Neue Zürcher Zeitung, 19. März 2007.

2 Kohler HP. Notfalldienst durch Grundversorger: Wie lange noch und durch wen? Schweiz Medizin Forum. 2006;6(25):597-8.
3 Salvage J, Smith R. Doctors and nurses: doing it differently. BMJ. 2000;320:1019-20.

4 Ormond-Walshe SE, Newham R. Comparing and contrasting the clinical nurse specialist and the advanced nurse practitioner roles. J Nurs Manag. 2001;9(4):205-7.

5 Leutenegger M. Pflegeberufe: Wenn Pfleger/innen den Doktor machen. Futura Bildungsmagazin. 2007;8(2):5-7.

6 Iankova A. The accountability of emergency nurse practitioners. Emerg Nurse. 2006;14(6):20-5.

7 Kesselring A. Interprofessionelle Zusammenarbeit zwischen Medizin und Pflege. Pflege. 2005;18(3): 143-5.

8 Spirig R, De Geest S. Advanced Nursing Practice lohnt sich! Pflege. 2004;17(4):233-6.

9 Nissen-Druey C. Akademische Pflege aus Ärztesicht. Managed Care. 2005;6:16-8.

10 Horrocks S, Anderson E, Salibury C. Systematic review of whether nurse practitioners working in primary care can provide equivalent care to doctors. BMJ. 2002;324:819-23.

11 Jenkins-Clarke S, Carr-Hill R. Changes, challenges and choices for the primary health care workforce: looking to the future. J Adv Nurs. 2001;34(6):842-9.

12 Flanagan L. Nurse practitioners: growing competition for family physicians? Family Practice Management. 1998;5(9):34-6, 41-3.

13 Venning P, Durie A, Roland M, Roberts C, Leese B. Randomised controlled trial comparing cost effectiveness of general practitioners and nurse practitioners in primary care. BMJ. 2000;320:1048-53.

14 Phillips RL, Harper DC, Wakefield M, Green LA, Fryer G. Can nurse practitioners and physicians beat parochialism into plowshares? Health Aff (Millwood). 2002;21(5):133-42.

15 Wilson A, Pearson D, Hassey A. Barriers to developing the nurse practitioner role in primary care the GP perspective. Fam Pract. 2002;19(6):641-6.

16 Ketefian S, Redman RW, Hanucharurnkul S, Masterson A, Neves EP. The development of advanced practice roles: implications in the international nursing community. Int Nurs Rev. 2001;48(3):152-63. 